\title{
Monotonicity Properties of the Family of Trapezoidal Maps
}

\author{
K. M. Brucks ${ }^{1 \star}$, M. Misiurewicz ${ }^{2 \star \star}$ and C. Tresser ${ }^{3}$ \\ 1 Institute for Mathematical Sciences, SUNY at Stony Brook, Stony Brook NY 11794-3660, USA \\ 2 The Institute for Advanced Study, School of Mathematics, Princeton NJ 08540, USA \\ 3 I. B. M, Thomas J. Watson Research Center, P. O. Box 218, Yorktown Heights NY 10598, USA
}

Received April 21, 1989; in revised form August 10, 1990

Abstract. Trapezoidal maps which are everywhere expanding out of their plateau form a three parameter family $T$, up to affine changes of coordinates. We show that splitting $T$ according to the various possible dynamical "behaviors" (we make this word precise in the process), yields a codimension one foliation. Some consequences of our result in terms of the monotonicity along simple one parameter families in $T$ are then drawn. All together, aperiodic behavior is rare both from the topological and the measure theoretical point of view in $T$.

\section{Introduction and Statement of the Results}

In this paper, we consider the set $T$ of "trapezoidal maps" (i.e., maps whose graph is trapezoidal) on the unit interval $I=[0,1]$, which absolute values of the slopes on both sides of the plateau greater than one, and which send both endpoints of I to 0 . These maps have been the object of many publications (see Sect. III), and it turns out that most of the questions about the evolution of the topological dynamics in one parameter families of such maps (see Theorem 1), and a related measure theoretical question (see Theorem 2) can be easily deduced from kneading theory $[4,8,9]$ and the following well known:

Main Fact. For a map in $T$, the Lebesgue measure of the union of all inverse images of the plateau $\mathscr{P}$ is one, i.e., $\lambda\left(\bigcup_{n=0}^{\infty} f^{-n}(\mathscr{P})\right)=1$.

For completeness a proof of this statement will be provided in the Appendix.

* University of Wisconsin-Milwaukee, Department of Mathematical Sciences, Milwaukee, WI 53201, USA

$\star \star$ Permanent Address: Instytut Matematyki, Uniwersytet Warszawski, Palac Kultury i Nauki IX p., PL-0-901 Warsaw, Poland 
We now need a few simple remarks and definitions to state our main results.

First, we can represent any $f$ in $T$ by a triple $(X, Y, Z)$, where $X$ stand stands for the inverse of the left slope, $Y$ for minus the inverse of the right slope, and $Z$ for the length of the plateau. Then $T$ is the set of all maps $f(x)=f_{(X, Y, Z)}(x)$ such that:

$$
(X, Y, Z) \in(0,1)^{3} \text { and } X+Y+Z \geqq 1 .
$$

$T$ is a metric space with the distance inherited from the usual distance in $\mathbb{R}^{3}$.

Set,

$$
M=\frac{1-Z}{X+Y}, \quad a=M X=\frac{X-Y Z}{X+Y}, \quad b=1-M Y=\frac{X+Y Z}{X+Y},
$$

and thus $\mathscr{P}=[a, b]$. Then,

$$
f(x)=f_{(X, Y, Z)}(x)= \begin{cases}x / X & \text { if } 0 \leqq x \leqq a, \\ M & \text { if } a \leqq x \leqq b, \\ (1-x) / Y & \text { if } b \leqq x \leqq 1\end{cases}
$$

Any point $x \in \mathscr{P}$, has an intrinsic coordinate $t=t(x)$ which is zero at the left-hand end $a$ of the plateau, one at the right-hand end $b$, and more generally is given by $t(x)=\frac{x-a}{b-a}$.

The last ingredient we will need is a bit of kneading theory $[4,8,9]$ adapted to trapezoidal maps. We will follow as much as possible the notations and conventions of [4]. Although this discussion is not quite standard, some readers might skip it at first reading, and go to the statement of the results. We distinguish between the periodic and aperiodic cases according to whether the maximal value $M$ is periodic or not.

The main specific feature of trapezoidal maps is that there are two ways to define addresses of points, each of them being significantly better in some context. We shall thus use both of them and speak of either $C$-kneading theory, with $C$-addresses, $C$-kneading sequences , . ., or of $P$-kneading theory, with $P$-addresses, $P$-kneading sequences $\cdots$. . In both cases, the address $a(x)$ of a point to the left of the plateau is $L$, while $a(x)$ is $R$ if $x$ lies to the right of the plateau. The itinerary $I(x)$ of $x$ is the sequence $(a(x), a(f(x)), \ldots)$; this sequence is infinite if it contains only $L$ 's and $R$ 's, and stops after the first $C$ or $P$. The kneading sequence $K(f)$ of $f$ is defined as the itinerary of $M$. With $Q$ standing for either $C$ or $P$, we set $L<Q<R$, and for two words in $L, Q, R$ beginning by $W A$ and $W B$ with $A<B$, we say that $W A \cdots<W B \cdots$ if $W$ contains an even number of $R$ 's, and that $W A \cdots>W B \cdots$ otherwise. We say that a sequence $S$, which is infinite if it contains only $L$ 's and $R$ 's, and stops after the first $C$ or $P$, is a unimodal kneading sequence if it can be realized as the kneading sequence of a unimodal map. This is equivalent to say that $S$ is not smaller than any of its right shifts.

- In the $C$-kneading language, we will assign $C$ as the address of the point $x_{0}$ in the plateau with intrinsic coordinate $t\left(x_{0}\right)=1 / 2$, and $a(x)=L$ for $x$ on the left of $x_{0}, a(x)=R$ for $x$ on the right of $x_{0}$. The consequence is that a same kneading sequence can correspond to two different periods for the stable periodic orbit in the periodic case. The $C$-kneading language is particularly adequate for the 
formulation of Theorem 1 and for the proof of Theorem 3. It is also the language we refer to when making comparisons with the smooth case.

- In the $P$-kneading language, we will assign $P$ as the address of all points in the plateau. Consequently the same kneading sequence can no longer correspond to two different periods. The price we pay is that periodic kneading sequences cannot be realized by trapezoidal maps in $T$ : in the periodic case, the kneading sequence is finite and terminates by $P$. The $P$-kneading language is particularly adequate for the statement of Lemma 1.

Remark 1. It is clear that an a periodic behavior corresponds to the same sequence in $C$ or $P$ kneading theory. Translating between the triple ( $C$-kneading sequence, period, $t)$ and the pair $(P$-kneading sequence, $t)$ corresponding to the same periodic behavior is also easy. However, having both languages at our disposal will allow shorter AND clearer statements.

Given two maps $f$ and $g$ in $T$ whose $P$-kneading sequences read respectively $K(f)$ and $K(g)$, we will say that the behavior $\mathscr{B}(f)$ of $f$ is smaller than the behavior $\mathscr{B}(g)$ of $g$ if either $K(f)<K(g)$, or $f$ and $g$ are periodic, $K(f)=K(g)$ and $t(f)<t(g)$ or $t(f)>t(g)$ according to whether the kneading sequence contains an even or an odd number of $R$ 's. (Here $t(f)$ stands for the intrinsic coordinate of the point in the plateau which is periodic with respect to the map $f$.) Hereby, we have implicitly defined the behavior $\mathscr{B}(f)$ of any map in $T$ which, according to Remark 1 , can also be described using the $C$-kneading language.

Remark 2. In the case of a smooth map, a behavior would be defined similarly in the aperiodic case, while the third member of the triple $(C$-kneading sequence, period, $t$ ) could be chosen as the multiplicator (or Lyapunov exponent) of the attracting periodic orbit in the periodic case.

Since this paper deals mainly with monotonicity properties, let us be precise on this matter. The word "strictly" will always mean that $x \neq y$ implies that $x$ and $y$ have different images under the map whose monotonicity we consider, while this implication will not be assumed to hold when "strictly" is not employed.

Theorem 1. The partition $\mathscr{F}$ of the space $T$ into the sets of maps with the same behavior is a codimension one foliation of $T$. Each leaf is a graph in the direction of any of the coordinates $X, Y, Z$. Moreover, the behavior strictly decreases with each of $X, Y, Z$.

Any unimodal $C$-kneading sequence is represented by a leaf of $\mathscr{F}$ in the aperiodic case, and by a connected continuum of leaves in the periodic case.

Let $T^{\prime}$ be the set of triangular maps (i.e., maps whose graph has a triangular convex envelope) on the unit interval $I$, with absolute values of both slopes greater than one. It is the $Z=0$ part of the boundary of $T$. The parameters $X$ and $Y$ provide natural coordinates for $T^{\prime}$. We cannot extend $\mathscr{F}$ to a foliation of $\bar{T}=T \cup T^{\prime}$, since bunches of leaves would collapse, due to the non-existence for such maps of kneading sequences which are not primary, (a $C$-kneading sequence $K$ is primary if it is larger than $R^{* \infty}$ and if $K=A^{*} B$ with $A \neq \varnothing, B \neq C$ implies that $A=R^{* m}$ for some $m$ ) [12]. However, these are the only collapsed bunches of leaves $[11,12]$.

This observation leads to the following corollary of Theorem 1 .

Corollary 1. The partition $\mathscr{S}$ of the space $T^{\prime}$ into the sets of maps with the same behavior is a stratification of $T^{\prime}$. Each component of the boundary of 
each stratum inside $T^{\prime}$ is a graph in the direction of any of the coordinates $X$ and $Y$. Moreover, the kneading sequence decreases with each of $X$ and $Y$.

Any primary unimodal $C$-kneading sequence is represented by a stratum of $\mathscr{S}$.

Remark 3. In fact, more is proved in [12], namely $\mathscr{S}$ is a foliation. We cannot get this foliation from our Theorem 1 without duplicating the estimates in [12]. We have reported Corollary 1 merely as an illustration of possible implications of the methods of this paper in other contexts.

Corollary 2. The topological entropy, as a function defined in $\bar{T}$, is decreasing with any of the parameters $X, Y, Z$.

Theorem 2. For any pair $(X, Y)$, the set of $Z$ values such that $f_{(X, Y, Z)}$ is aperiodic has Lebesgue measure zero.

Corollary 3. The union of all aperiodic leaves in $\mathscr{F}$ has zero (three-dimensional) Lebesgue measure.

We conjecture that the foliation $\mathscr{F}$ in Theorem 1 is smooth. However the only thing we can prove is the following:

Proposition. For all leaves of $\mathscr{F}$ corresponding to a periodic behavior and $t=0$ or $t=1$, there are estimates from above on $\frac{d Z}{d X}$ and $\frac{d Z}{d Y}$ by positive continuous functions of $(X, Y, Z)$, independent of the particular behavior.

Proof of the Proposition. Let $\lambda=1 / X, \mu=1 / Y$. For a fixed $x$, denote

$$
a_{n}=\frac{\partial f^{n}(x)}{\partial \lambda}, \quad b_{n}=\frac{\partial f^{n}(x)}{\partial \mu}
$$

Then by an easy induction, we get that if among $a(x), a(f(x)), \ldots, a\left(f^{n-1}(x)\right)$ there are $k L$ 's and $m R$ 's $(k+m=n)$, then

$$
\left|a_{n}\right| \leqq \frac{\lambda^{k} \mu^{m}-1}{\lambda-1},\left|b_{n}\right| \leqq \frac{\lambda^{k} \mu^{m}-1}{\mu-1} .
$$

If we fix $\mu$ and $Z$, and let $\lambda=\lambda(t), x=x(t)$ and $f^{n}(x) \equiv x$ (with $k L$ 's and $m R$ 's as above), then we get $\left|\frac{d x}{d y}\right| \leqq \frac{1}{\lambda-1}$. Analogously, if we fix $\lambda$ and $Z$, and let $\mu=\mu(t), x=x(t)$ and $f^{n}(x) \equiv x$ then $\left|\frac{d x}{d \mu}\right| \leqq \frac{1}{\mu-1}$. Now if ve look at the leaves of $\mathscr{F}$ corresponding to a periodic behavior and $t=0$ or $t=1$, then $\lambda=1 / X, \mu=1 / Y$ and either $x=a=\frac{X-Y Z}{X+Y}$, or $x=b=\frac{X+Y Z}{X+Y}$.

In both cases we get the desired estimates by easy calculations.

(Proposition)

Corollary 4. For all leaves of $\mathscr{F}$ corresponding either to a periodic behavior and $t=0$ or $t=1$, or to aperiodic behavior, $Z$ is a locally Lipschitz continuous function of $X$ and $Y$ and the Lipschitz constant is uniformly bounded on compact subsets of $T$. 


\section{Proofs of the Theorems}

Proof of Theoerm 1. The following result is (like the above mentioned "Main Fact") part of the folklore. Because we could not find a satisfactory reference, we formalize it as:

\section{Lemma 1 and Definition (Preimages of the plateau).}

i) In the aperiodic case, any component of the preimage $f^{-n}(\mathscr{P})$ has length $U_{1} \cdot U_{2} \cdots \cdot U_{n} \cdot Z$, where $U_{i}=X$ if the $i^{\text {th }}$ preimage is taken on the left-hand side, and $U_{i}=Y$ otherwise. Furthermore, for each point $x$ whose orbit intersects the plateau there is a unique $n \geqq 0$ and a unique component of $f^{-n}(\mathscr{P})$ such that $x$ belongs to this component.

ii) In the periodic case, when the point in the plateau with intrinsic coordinate $t$ has period $p$, the plateau is contained in the $k^{\text {th }}$ preimage of itself, for all $k$ which are multiples of $p$. To avoid dealing with possible intersections of preimages, we recognize that the plateau is the unique component of $f^{-0}(\mathscr{P})$, and for $n>0$, we only consider preimages on the left or on the right, i.e., never in the plateau. With this modified definition, two components of the preimage $f^{-n}(\mathscr{P})$ have length $m \cdot U_{1} \cdot U_{2} \cdots \cdot U_{n} \cdot Z$, where $m$ stands for $t$ or $1-t$ according to whether there is an even or an odd number of R's in the P-kneading sequence of the map. Any preimages of these components will carry along this factor $m$, the length of all other components of any preimage being computed like in the aperiodic case. Furthermore, with this modified definition of a component of a preimage, we can ensure again that for each point $x$ whose orbit intersects the plateau, there is a unique $n \geqq 0$ and a unique component of $f^{-n}(\mathscr{P})$ such that $x$ belongs to this component.

The proof is obvious and left to the reader (see Fig. 1).

In order to correctly use the information contained in Lemma 1, we will need some facts which are just translations of known results in kneading theory $[4,8,9]$, collected here under the following:

Remark 2. Assume that the $P$-kneading sequence increases, then:

- no inverse legal path (i.e., sequence of rights and lefts allowed for taking a preimage of the plateau [8]) can disappear, but new ones do appear, - any inverse path which would carry the $m$ factor for the smaller $P$-kneading sequence, looses this factor for the bigger one,

- if an inverse path carries the $m$ factor for the bigger $P$-kneading sequence, this path is not a legal inverse path for the smaller one.

After putting all this together, we arrive at the first new fact:

Lemma 2. For any one parameter family $\left\{f_{C}\right\}_{C}$ with

$$
(A, B) \in\{(X, Y),(X, Z),(Y, Z)\}
$$

and where $C$ stands for the third coordinate in $T, C_{1}>C_{2}$ implies that $\mathscr{B}\left(f_{C_{1}}\right)<\mathscr{B}\left(f_{C_{2}}\right)$.

Proof of Lemma 2. Assume on the contrary that $\mathscr{B}\left(f_{C_{1}}\right) \geqq \mathscr{B}\left(f_{C_{2}}\right)$. Then from what we know about preimages of the plateau (Lemma 1 and Remark 2), it follows that for each $n>0$ :

$$
\lambda\left(f_{C_{1}}^{-n}(\mathscr{P})\right)>\lambda\left(f_{C_{2}}^{-n}(\mathscr{P})\right) .
$$

This contradicts the Main Fact.

$$
\text { (Lemma 2) }
$$




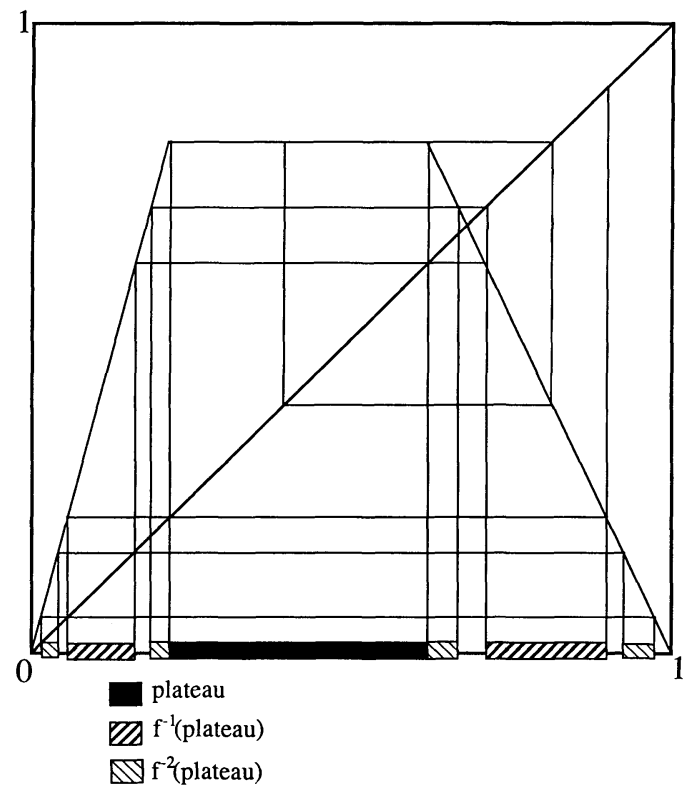

Fig. 1.

We remark that Lemma 2 proves that the partition $\mathscr{F}$ given in Theorem 1 has the monotonicity properties claimed in Theorem 1 . We now proceed to prove that $\mathscr{F}$ is indeed a codimension one foliation. For convenience, elements of the partition $\mathscr{F}$ are denoted with $\mathscr{L}$ 's. We need three further known (and easy) facts stated here as Lemmas 3,4, and 5.

Lemma 3. Suppose that the maps $f_{\left(X, Y, Z_{0}\right)}, f_{\left(X, Y, Z_{1}\right)} \in T$ have kneading sequences $K_{0}$ and $K_{1}$ respectively. Then any unimodal $C$-kneading sequence between $K_{0}$ and $K_{1}$ is realized by some $f_{(X, Y, Z)}$ with $Z$ between $Z_{0}$ and $Z_{1}$.

Lemma 3 can be easily deduced from Theorem 3 below. It could also be proven along the lines of Theorem 5.2 of [1], or of Theorem II.3.8 of [4].

Lemma 4. Finite $C$-kneading sequences are dense in the set of unimodal C-kneading sequences.

Lemma 5. For any given finite P-kneading sequence, the set $U$ of $(X, Y, Z)$ 's for which $f_{(X, Y, Z)}$ has this kneading sequence with $t \in(0,1)$ is open. In $U$, the dependence of $t$ on $(X, Y, Z)$ is continuous. Moreover, as $(X, Y, Z)$ approaches the boundary of $U$, then either $f_{(X, Y, Z)}$ approaches the boundary of $T$ or $t$ approaches 0 or 1 .

The following corollary is an intermediate value statement for behaviors, which follows from Lemmas 3 and 5.

Corollary 6. Suppose that the maps $f_{\left(X, Y, Z_{0}\right)}, f_{\left(X, Y, Z_{1}\right)} \in T$ have behaviors $\mathscr{B}_{0}$ and $\mathscr{B}_{1}$ respectively. Then any behavior between $\mathscr{B}_{0}$ and $\mathscr{B}_{1}$ is realized by some $f_{(X, Y, Z)}$ with $Z$ between $Z_{0}$ and $Z_{1}$. 
We point out that $T$ was defined as a set of functions, not points of $(0,1)^{3}$. However, for convenience we will sometimes use " $(X, Y, Z) \in T$ " in place of " $f_{(X, Y, Z)} \in T$ ".

We next remark that each $\mathscr{L} \in \mathscr{F}$ is the graph of some function $Z=\Phi_{\mathscr{L}}(X, Y)$. This follows immediately from Lemma 2. The next lemma proves that $\Phi_{\mathscr{L}}$ is continuous. Let $U_{\mathscr{L}}$ deote the domain of $\Phi_{\mathscr{L}}$.

Lemma 7. Let $\mathscr{L} \in \mathscr{F}$. Then $\Phi_{\mathscr{L}}$ is continuous.

Proof of Lemma 7. We break the proof into two parts. First assume that $\mathscr{L}$ corresponds to a periodic behavior with $t \in(0,1)$. We will show that

(*) $U_{\mathscr{L}}$ is open and $\Phi_{\mathscr{L}}$ is continuous.

Fix a point $(X, Y, Z) \in \mathscr{L}$. We will use the notation $t(\cdot, \cdot, \cdot)$ to indicate the intrinsic coordinate $t$ of a map $f_{(\cdot,,, \cdot)}$ cooresponding to a point in the set $U$ from Lemma 5. By Lemma 2, on each vertical line (the axis $Z$ is vertical) in $U$, the $t$ 's are ordered in the same monotone way. Now we use Lemma 5 . On the vertical line through $(X, Y, Z)$ there are points $\left(X, Y, Z_{1}\right)$ and $\left(X, Y, Z_{2}\right)$ on both sides of $(X, Y, Z)$ with $t\left(X, Y, Z_{i}\right) \in(0,1)$ for $i=1,2$ and $t\left(X, Y, Z_{1}\right)<t(X, Y, Z)<t\left(X, Y, Z_{2}\right)$. By the continuity of $t$, there is a neighborhood $V$ of $(X, Y)$ such that for $\left(X^{\prime}, Y^{\prime}\right) \in V$, we have $t\left(X^{\prime}, Y^{\prime}, Z_{1}\right)<t(X, Y, Z)<t\left(X^{\prime}, Y^{\prime}, Z_{2}\right)$. Therefore, by the continuity of $t$, there is some $Z^{\prime}$ between $Z_{1}$ and $Z_{2}$ with $t\left(X^{\prime}, Y^{\prime}, Z^{\prime}\right)=t(X, Y, Z)$; that is, $\left(X^{\prime}, Y^{\prime}, Z^{\prime}\right) \in \mathscr{L}$. This proves that $V \subset U_{\mathscr{L}}$, and thus $U_{\mathscr{L}}$ is open. Moreover, since we could take $Z_{1}$ and $Z_{2}$ arbitrarily close to $Z$, this also proves the continuity of $\Phi_{\mathscr{L}}$. Thus, we have proven $(*)$.

We now need to prove that $\Phi_{\mathscr{L}}$ is continuous for an arbitrary $\mathscr{L}$. The argument is similar to the above. If $\mathscr{L} \in \mathscr{F}$ is arbitrary and $(X, Y, Z) \in \mathscr{L}$, then, in view of Lemma 4, we can find $Z_{1}$ and $Z_{2}$ arbitrarily close to $Z$ and such that $Z_{1}<Z<Z_{2}$, $\left(X, Y, Z_{1}\right) \in \mathscr{L}_{1},\left(X, Y, Z_{2}\right) \in \mathscr{L}_{2}$, and $\mathscr{L}_{1}, \mathscr{L}_{2}$ correspond to periodic $C$-kneading sequences. Then the continuity of $\Phi_{\mathscr{L}}$ follows as in the proof of $(*)$, using Lemma 2 and (in the place of the continuity of $t)(*)$. Note that this does not work if $X+Y+Z=1$, since we cannot choose $Z_{1}$. However, in this case $\mathscr{L}=\{(X, Y, Z) \in$ $\left.(0,1)^{3} \mid X+Y+Z=1\right\}$. $\quad$ (Lemma 7)

Now notice that for any $\mathscr{L} \in \mathscr{F}$, the set $U_{\mathscr{L}}$ contains with each point $(X, Y)$ the entire rectangle $(0, X] \times(0, Y]$. To see this, choose $0<\tilde{X}<X$ and $0<\tilde{Y}<Y$, and let $Z=\Phi_{\mathscr{L}}(X, Y)$. If $(\tilde{X}, \tilde{Y}, Z)$ is in $T$, then, by Lemma $2, \mathscr{B}\left(f_{X, Y, Z}\right)<\mathscr{B}\left(f_{\tilde{X}, \tilde{Y}, Z}\right)$ and hence, by Corollary $6,(\tilde{X}, \tilde{Y})$ is in the domain of $\Phi_{\mathscr{L}}$. If $(\tilde{X}, \tilde{Y}, Z)$ is not in $T$, then there is some $\tilde{Z} \in(0,1)$ such that $\tilde{X}+\widetilde{Y}+\widetilde{Z}=1$, i.e., $M=1$ (for the map $f_{\tilde{X}, \tilde{Y}, \tilde{Z}}$ ). Again, by Corollary $6,(\tilde{X}, \widetilde{Y})$ is in the domain of $\Phi_{\mathscr{L}}$.

We can now conclude the proof of Theorem 1:

For any $\left(X, Y, Z_{1}\right),\left(X, Y, Z_{2}\right) \in T$ with $Z_{1}<Z_{2}$, the set $\left\{\left(X^{\prime}, Y^{\prime}, Z^{\prime}\right) \in T \mid X^{\prime} \leqq X\right.$, $\left.Y^{\prime} \leqq Y, \mathscr{B}\left(X, Y, Z_{1}\right) \geqq \mathscr{B}\left(X^{\prime}, Y^{\prime}, Z^{\prime}\right) \geqq \mathscr{B}\left(X, Y, Z_{2}\right)\right\}$ admits, by the previous paragraph and Lemma 2 , the coordinate system $\left(X^{\prime}, Y^{\prime}, \mathscr{B}\left(X^{\prime}, Y^{\prime}, Z^{\prime}\right)\right)$. The coordinate change is continuous by Lemmas 2 and 7 . This completes the proof of Theorem 1.

- (Theorem 1)

Proof of Theorem 2 and Lemma 3. Theorem 2, as well as Lemma 3, is a particular case of the following (see [6]): 
Theorem 3. Let $f_{1}:[0,1] \rightarrow[0,1]$ with $f(0)=f(1)=0$ be a map with a single interval $J$ (perhaps reduced to a point) in $(0,1)$, where $f(x)$ attains its maximal value, and $f$ increasing on the left of $J$, decreasing on the right of $J$. Define the cutting out family $\left\{f_{R}\right\}_{0 \leqq R} \leqq$ of $f_{1}$ by:

$$
f_{R}(x)=\min \left(f_{1}(x), R\right) .
$$

Then,

i) Any unimodal kneading sequence not greater than $K\left(f_{1}\right)$ is realized by some $f_{R}$ with $0 \leqq R \leqq 1$.

ii) $R_{1}>R_{2}$ implies that $K\left(f_{R_{1}}\right) \geqq K\left(f_{R_{2}}\right)$.

iii) Furthermore, if $f_{1}$ has no homterval out of $\bigcup_{n \geqq 0} f_{1}^{-n}(J)$, then any unimodal kneading sequence not greater than $K\left(f_{1}\right)$, and corresponding either to an a periodic behavior, or to a periodic behavior with $t=0$ or $t=1$ is realized by a single $f_{R}$ with $0 \leqq R \leqq 1$.

iv) Furthermore, if $f_{1}$

a) has everywhere the absolute value of its slope greater than 1, except perhaps on a plateau where it takes its maximal value,

$\beta$ ) or has a negative Schwarzian derivative everywhere, except perhaps on a plateau where its takes its maximum value,

then, $\lambda\left\{R \mid f_{R}\right.$ has no stable periodic orbit $\}=0$.

Proof of Theorem 3. The basic fact in cutting out families is that any kneading sequence for a $f_{R}$ can be read as an itinerary of some point under $f_{1}$, these points being precisely those which bound their orbit from above. This takes care of statements i), ii), and iii).

For statement iv), the case $\alpha$ ) with a plateau is covered by Theorem 4 in the Appendix, and the case without a plateau is then a simple consequence, since by cutting an arbitrarily small piece of the tip, one gets a plateau. That the case $\beta$ ) behaves like the case $\alpha$ ) is a result of [10]. Details are left to the reader.

(Theorems 2 and 3, and Lemma 3)

\section{Some Remarks}

As we already mentioned, Corollary 1 was already known in a stronger form [12]. It corresponds to the face $Z=0$ of $T$, i.e., to triangular maps. We refer to [12] for more historical background in this context.

As far as proper trapezoidal maps are concerned, most previous studies were devoted to the subspace $X=Y$ of $T$ (i.e., maps with a symmetry), and more precisely to one parameter families with a constant length $Z$ of the plateau. We remark that our Lemma 2 works as well for such families. Almost everything was known for this particular case of our Theorem 1, except for the periodic case with $t \neq 1 / 2$. While a short proof was available in the periodic case with $t=1 / 2$ for $Z>(13-3 \sqrt{17}) / 2[1]$, the only general result for $X=Y$ was through quite a long proof [7], on which relied the short treatment of the aperiodic case in [3]. Also a proof was provided in [5] for the particular case $X=Y=1 / 2$ of our Theorem 2 .

The main motivation for results such as our Theorem 1 is that getting similar results for the quadratic family would be quite important. Presently, only periodic 
behavior and exceptional types of aperiodic behavior are understood at the level of Theorem 1 for $1-a x^{2}$ (see e.g., [6] for recent results and more history), most of the proofs being quite difficult. Hence our results provide a model for the conjectured evolution of the dynamics in the quadratic family, and may be a new way to think about it, if not a path for proofs.

Conjecture. If for a one-parameter family $\left\{f_{t}\right\}$ of piecewise smooth unimodal maps, $\Sigma \partial_{t} P^{n} f_{t}(1)(c)<0$ (perhaps $=-\infty$ ), where $P f_{t}$ is the Perron-Frobenius operator for $f_{t}$ and $c$ is the common critical point of all $f_{t}$,s, then $K\left(f_{t}\right)$ is increasing in $t$.

If true, this conjecture could provide a tool for proving monotonicity for some one-parameter families of unimodal maps.

Theorem 2 is less relevant for smooth dynamics since one knows that for ergodic properties, the quadratic family behaves quite differently.

Acknowledgements. M.M. thanks the I.A.S. for an invitation for the second term of the academic year 1988-1989. C.T. thanks the Arizona Center for Mathematical Sciences (ACMS) for an invitation for the academic year 1988-1989. The ACMS is sponsored by AFOSR contract 49620-86-c0130 with the URI program at the University of Arizona. All three authors express their gratitude to A. G. Bell for inventing the telephone which was instrumental in the completion of the present work.

\section{Appendix}

The "Main Fact" which serves as a basis of our results could be extracted, for instance, from [10], and is nothing but a particular case of the following:

Theorem 4. Let $0<a<b<1$ and let $f:[0,1] \rightarrow[0,1]$ be a map such that on $[0, a]$ and $[b, 1], f$ is of class $C^{2}$ and

$$
\inf \left\{\left|f^{\prime}(x)\right|: x \in[0, a] \cup[b, 1]\right\}>1 .
$$

Then the Lebesgue measure

$$
\lambda\left(\left\{x \in[0,1] \mid f^{n}(x) \notin(a, b) \text { for } n=0,1,2, \ldots\right\}\right)=0 .
$$

Proof. We can find points $c, d$ such that $a<c<d<b$ and a map $g: A \rightarrow[0,1]$, where $A=[0, c] \cup[d, 1]$, such that (see Fig. 2):

$-g=f$ on $[0, a] \cup[b, 1]$,

$-g$ is of class $C^{2}$ on each of $[0, c]$ and $[d, 1]$,

$-g$ maps each of $[0, c]$ and $[d, 1]$ onto $[0,1]$,

$-\inf _{x \in A}\left|g^{\prime}(x)\right|=\alpha>1$.

For each $n$, the set

$$
\begin{aligned}
D_{n} & =\left\{x \in[0,1] \mid f^{i}(x) \notin(a, b) \text { for } i=0,1, \ldots, n-1\right\} \\
& =\left\{x \in[0,1] \mid g^{i}(x) \notin(a, b) \text { for } i=0,1, \ldots, n-1\right\},
\end{aligned}
$$

is contained in the set

$$
E_{n}=\left\{x \in[0,1] \mid g^{i}(x) \in A \text { for } i=0,1, \ldots, n-1\right\} .
$$




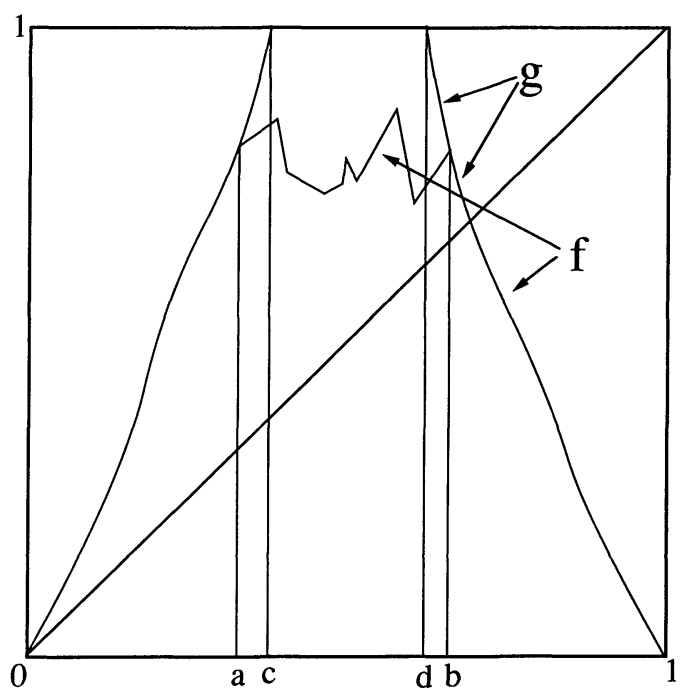

Fig. 2.

Our aim is to show that $\lambda\left(\bigcap_{n=0}^{\infty} D_{n}\right)=0$. In fact we will show that even $\lambda\left(\bigcap_{n=0}^{\infty} E_{n}\right)=0$.

Notice that $E_{n}$ is the domain of $g^{n}$. One can easily see by induction that $E_{n}$ is a union of $2^{n}$ disjoint intervals $\Delta_{k, n}\left(k=0,1, \ldots, 2^{n}-1\right)$ and each of them is mapped onto $[0,1]$ by $g^{n}$. Then the set $E_{n+1}$ is obtained from $E_{n}$ by taking each $\Delta_{k, n}$ and dividing it into three subintervals:

$$
\left(\left.g^{n}\right|_{\Delta_{k, n}}\right)^{-1}([0, c]),\left(\left.g^{n}\right|_{\Delta_{k, n}}\right)^{-1}([d, 1]) \text {, and }\left(\left.g^{n}\right|_{\Delta_{k, n}}\right)^{-1}([c, d]) \text {, }
$$

where the first two of these intervals are the components of $E_{n+1}$ contained in $E_{n}$, and the third one is disjoint from $E_{n+1}$. To compare their measures, we have to estimate the distortion of $g^{n}$, i.e., the maximal possible ratio of derivatives of $g^{n}$ on $\Delta_{k, n}$.

Suppose that $x, y \in \Delta_{k, n}$. The constant:

$$
\beta=\sup \left|\left(\log \left|g^{\prime}(z)\right|\right)^{\prime}\right|=\sup \left|\frac{g^{\prime \prime}(z)}{g^{\prime}(z)}\right|
$$

is finite since $g$ is of class $C^{2}$. We have:

$$
\begin{gathered}
|\log |\left(g^{n}\right)^{\prime}(x)|-\log |\left(g^{n}\right)^{\prime}(y)||=\left|\sum_{i=0}^{n-1} \log \right| g^{\prime}\left(g^{i}(x)\right)\left|-\sum_{i=0}^{n-1} \log \right| g^{\prime}\left(g^{i}(y)\right)|| \\
\leqq \sum_{i=0}^{n-1}|\log | g^{\prime}\left(g^{i}(x)\right)|-\log | g^{\prime}\left(g^{i}(y)\right)|| \leqq \sum_{i=0}^{n-1} \beta \cdot\left|g^{i}(x)-g^{i}(y)\right|,
\end{gathered}
$$

since $g^{i}(x)$ and $g^{i}(y)$ lie in the same component of $A$. We have also:

$$
1 \geqq\left|g^{n}(x)-g^{n}(y)\right| \geqq \alpha^{n-i} \cdot\left|\geqq \alpha^{n-i} \cdot\right| g^{i}(x)-g^{i}(y) \mid
$$


thus,

$$
|\log |\left(g^{n}\right)^{\prime}(x)|-\log |\left(g^{n}\right)^{\prime}(y)|| \leqq \beta \cdot \sum_{i=0}^{n-1} \alpha^{i-n}<\beta \cdot \sum_{j=1}^{\infty} \alpha^{-j}
$$
Since $\alpha>1$, the constant $\gamma=\exp \left(\beta \cdot \sum_{j=1}^{\infty} \alpha^{-j}\right)$ is finite and we get the following
estimates for the distortion:

$$
\sup _{x, y \in \Delta_{k, n}}\left|\frac{\left(g^{n}\right)^{\prime}(x)}{\left(g^{n}\right)^{\prime}(y)}\right| \leqq \gamma
$$

Consequently,

$$
\frac{\inf _{x \in \Delta_{k, n}}\left|\left(g^{n}\right)^{\prime}(x)\right|}{\sup _{x \in \Delta_{k, n}}\left|\left(g^{n}\right)^{\prime}(x)\right|} \geqq \frac{1}{\gamma} .
$$

Now if we denote $G_{k, n}=\left(\left.g^{n}\right|_{\Delta_{k, n}}\right)^{-1}((c, d))$, then:

$$
1=\int_{\Delta_{k, n}}\left|\left(g^{n}\right)^{\prime}(x)\right| d x \geqq \lambda\left(\Delta_{k, n}\right) \cdot \inf _{x \in \Delta_{k, n}}\left|\left(g^{n}\right)^{\prime}(x)\right|,
$$

and

$$
d-c=\int_{G_{k, n}}\left|\left(g^{n}\right)^{\prime}(x)\right| d x \leqq \lambda\left(G_{k, n}\right) \cdot \sup _{x \in G_{k, n}}\left|\left(g^{n}\right)^{\prime}(x)\right| .
$$

Consequently,

$$
\frac{\lambda\left(G_{k, n}\right)}{\lambda\left(\Delta_{k, n}\right)} \geqq \frac{d-c}{\gamma} .
$$

The above inequality holds for all $k$ and we get:

$$
\lambda\left(E_{n+1}\right) \leqq \lambda\left(E_{n}\right) \cdot\left(1-\frac{d-c}{\gamma}\right) .
$$

Hence, by induction, we get (since $E_{0}=[0,1]$ ):

$$
\lambda\left(E_{m}\right) \leqq\left(1-\frac{d-c}{\gamma}\right)^{m}
$$

so

$$
\lambda\left(\bigcap_{n=0}^{\infty} E_{n}\right) \leqq \lim _{m \rightarrow \infty}\left(1-\frac{d-c}{\gamma}\right)^{m}=0 .
$$

Remark 4. Theorem 4 is known to hold in $C^{1+\varepsilon}$. It is also known to be false in $C^{1}$ [2].

\section{References}

1. Beyer, W. A., Mauldin, R. D., Stein, P. R.: Shift-maximal sequences in function iteration: Existence, uniqueness, and multiplicity. J. Math. Anal. Appl. 115, 305-362 (1986)

2. Bowen, R.: A horsehoe with positive measure. Invent. Math. 29, 203-204 (1975)

3. Brucks, K. M.: Uniqueness of aperiodic kneading sequences. Proc. Am. Math. Soc. 107, 223-229 (1989) 
4. Collet, P., Eckmann, J.-P.: Iterated maps on the Interval as Dynamical Systems. Basel: Birkhäuser 1980

5. Derrida, B.: Critical properties of one dimensional mappings. In: Bifurcation Phenomena in mathematical physics and related topics. Bardos, C., Bessis, D. (eds.), Dordrecht: D. Reidel 1980

6. Gambaudo, J. M., Tresser, C.: A weak monotonicity property in one dimensional dynamics. Preprint U. of Arizona (1989)

7. Louck, J. D., Metropolis, N.: Symbolic dynamics of trapezoidal maps. Dordrecht: D. Reidel 1986

8. Metropolis, N., Stein, M. L., Stein, P. R.: On finite limit sets for transformations on the unit interval. J. Comb. Theory 15, 25-44 (1973)

9. Milnor, J., Thurston, W.: On interated maps of the interval. Lecture Notes in Mathematics, vol. 1342, pp. 465-563. Berlin, Heidelberg, New York: Springer 1988

10. Misiurewicz, M.: Absolutely continuous measures for certain maps of an interval. Publ. Math. I.H.E.S. 53, 17-52 (1981)

11. Misiurewicz, M.: Jumps of entropy in one dimension. Fund. Math. 132, 215-226 (1989)

12. Misiurewicz, M., Visinescu, E.: Kneading sequences of skew tent maps. Preprint (1987)

Communicated by J.-P. Eckmann 